\title{
A Walrasian Theory of Commodity Money: Paradoxical Results
}

\author{
Xavier Cuadras-Morató
}

Department of Economics

Universitat Pompeu Fabra

Ramon Trias Fargas, 25-27

08005 BARCELONA

e-mail: xavier.cuadras@econ.upf.es

JEL classification: D50, D82, E40

\begin{abstract}
Summary
The objective of this note is to analyze some implications of the model of commodity money described in Banerjee and Maskin (1996) which may seem paradoxical. In order to do this, we incorporate a general production cost structure into the model. We focus on two different results. First, the existence of technologies that make counterfeiting a commodity more difficult may exclude it from being used as medium of exchange. Second, allocative distortions due to problems of asymmetric information may become larger in the presence of such technologies.
\end{abstract}

* I am grateful to Andreu Mas-Colell, Eric S. Maskin, and an anonymous referee for helpful comments. All errors are my own. 


\section{Introduction}

Banerjee and Maskin (1996) (henceforth BM) describe an economy in which there is a unique commodity playing the role of money in equilibrium. In their model, all goods come in two different qualities (high quality goods and "lemons") which agents cannot generally distinguish. It turns out that the commodity used as medium of exchange in equilibrium is the one for which the discrepancy between qualities is smallest. This discrepancy is measured as the deviation of the marginal rate of substitution between consuming goods of high or low quality from one. BM claim that their model is a formal explanation of why gold (or other metals) has been widely used as commodity money through history. According to their interpretation, gold is a commodity for which the discrepancy between qualities that are undetectable is particularly small.

Commodities also vary on the amount of resources that must be spent to produce versions of different quality. Some goods are very easy to fake (that is, producing a low quality version which goes undetected is relatively cheap), while for some other commodities producing a "lemon" can be almost as expensive as producing a high quality good. How does this influence what commodity will be used as medium of exchange? Conventional economic wisdom suggests that goods that can be easily counterfeited are not likely to be used as media of exchange. So, the more expensive is to produce lemons relative to produce goods of high quality, the more likely will be a particular good to emerge as commodity money in equilibrium. 
The objective of this paper is to present some results in the context of the model of BM that might seem contradictory with the common intuition we mention above. In order to motivate the analysis, let us examine the following little example. Suppose that there are two almost identical economies that differ only in the technology available to produce undistinguishable low quality versions of a particular good (e.g. gold coins). ${ }^{1}$ In the first economy, this technology is primitive and, consequently, producing low quality coins that go undetected is relatively costly. In the second case the technology is more advanced, which means that producing the same fake gold coins is much cheaper. We prove in this note that, within the context of BM, it is more likely that gold coins circulate as medium of exchange in the second economy than in the first. Moreover, the distortions generated by the use of money (in terms of efficiency losses) to overcome informational constraints are higher in the first (with low cost of counterfeiting) economy. In the final part of the paper, we discuss the economic intuitions that lie behind these apparently paradoxical implications of the model.

\section{The Model}

The model we outline in the following paragraphs is basically the same as BM. The only difference is the production cost structure we assume, which is slightly more general than theirs. This allows us to present some interesting implications of the model.

There is a large, but finite, number of agents of type A, B, and C. These agents are specialized in production and consumption. Agents of type A $(B, C)$ produce only good 
A $(B, C)$ and consume only good B $(\mathrm{C}, \mathrm{A})$. These are the only three types of goods of the economy. There is not fiat money. To keep things symmetric, we will assume that there is an equal number of each type of agents. Given this pattern of specialization, trade is a necessary condition for consumption. Moreover, some form of monetary exchange has to emerge if there is going to be trade at all, since there is never double coincidence of wants between individuals who exchange the goods they produce. Goods are perfectly divisible and come in two different qualities: $\mathrm{X}_{1}$ and $\mathrm{X}_{2}(\mathrm{X} \in\{\mathrm{A}, \mathrm{B}, \mathrm{C}\})$.

Agents are all endowed with one unit of labor. Production costs are as follows: in order to produce a unit of a low quality good, $\mathrm{X}_{2}$, it is necessary to spend $1^{\mathrm{X}}$ units of labor; it takes $\mathrm{h}^{\mathrm{X}}$ units of labor to produce a unit of a high quality good, $\mathrm{X}_{1}\left(\mathrm{~h}^{\mathrm{X}}>1^{\mathrm{X}}\right)$. Obviously, $\mathrm{h}^{\mathrm{X}} / 1^{\mathrm{X}}$ is the marginal rate of transformation between high quality and low quality goods of type $\mathrm{X}\left(\mathrm{MRT}^{\mathrm{X}}\right)$. The closer is $\mathrm{MRT}^{\mathrm{X}}$ to one, the costlier is producing a "lemon" relatively to producing a high quality good. Consequently, the existence of technologies that make counterfeiting good $\mathrm{X}$ cheap will result in high values for $\mathrm{MRT}^{\mathrm{X}}$ (under our alternative interpretation, the worse the technology to detect "lemons", the higher the values for $\mathrm{MRT}^{\mathrm{X}}$ ).

Preferences of agents of type A can be described with the utility function $\mathrm{k}^{\mathrm{B}} \mathrm{b}_{1}+$ $b^{2}$, where $b^{i}$ is consumption of good $B^{i}$ and $k^{B}$ is a scalar. Preferences for agents of type $B$ and $\mathrm{C}$ can be described in a similar way. $\mathrm{k}^{\mathrm{X}}$ can be interpreted as the marginal rate of substitution between consuming a high or a low quality good of type $\mathrm{X}\left(\mathrm{MRS}^{\mathrm{X}}\right)$. The closer is $\mathrm{k}^{\mathrm{X}}$ to one, the smaller is the discrepancy between consuming goods of different quality. Assuming $\mathrm{k}^{\mathrm{X}}>\mathrm{MRT}^{\mathrm{X}}$ simply means that, in the absence of informational 
imperfections, it is inefficient to produce low quality goods.

Individuals in this economy are subject to the following informational constraint: a trader of type A can perfectly distinguish between goods $\mathrm{A}_{1}$ and $\mathrm{A}_{2}$ (production good) and $\mathrm{B}_{1}$ and $\mathrm{B}_{2}$ (consumption good), but cannot differentiate between $\mathrm{C}_{1}$ and $\mathrm{C}_{2}$ (rest of goods). The pattern of specialization of this economy ensures that traders of type A will only accept good $\mathrm{C}$ as means of obtaining some other good they can consume. Moreover, the informational restrictions mean that an A-trader will never be able to carry out any trade that involves good $\mathrm{C}_{1}$ (the argument generalizes immediately for agents of type $\mathrm{B}$ and $\mathrm{C}$ ). This implies that any commodity which is accepted not for immediate consumption and, hence, circulates as medium of exchange will necessarily be of low quality, which can be interpreted as a restatement of Gresham's law in the model.

There exist as many markets as goods are sold in this economy. Markets are spatially dispersed and there is not a centralized mechanism (e.g. clearinghouse) where exchanges can be executed. Trade is strictly bilateral which means that each exchange involves only two sides. We can also assume, without loss of generality (see BM, p.968), that transactions never involve more than two goods. Moreover, since there is not fiat money, commodities will be exchanged only for other commodities. Trade is also completely anonymous and unmonitorable by third parties. This means that there cannot be credit, futures contracts or short sales. For simplicity, we will assume that traders of the same type never exchange with each other. It can be shown easily that this assumption does not influence the aggregated equilibrium results (prices and production, trade, and consumption quantities). 
There is a finite number of discrete trading periods, T. This is important because, given the specialization pattern of agents in this model, reselling is necessary for consumption. Multiple trading periods obviously allow for this possibility. Agents can be conceived as performing two different roles in the economy: selling and buying. Each trading period, agents carry out at most two transactions corresponding to those roles. On the one hand, the buyer side can choose to visit (at most) one market in order to acquire the good sold there and, on the other hand, the seller side stays in the market which corresponds to his production good. Production takes place before the start of the first trading period. Any good that is not consumed before the end of period $\mathrm{T}$ disappears.

There are many sellers and buyers of a particular good in each market, so equilibrium will be competitive in the sense that agents will take prices as given. We will denote as $\mathrm{pt}\left(\mathrm{X}_{\mathrm{i}}, \mathrm{Y}_{\mathrm{j}}\right)$ the relative price of $\operatorname{good} \mathrm{X}^{\mathrm{i}}$ in terms of units of $\operatorname{good} \mathrm{Yj}_{\mathrm{j}}(\mathrm{X}, \mathrm{Y} \in$ $\{A, B, C\}, i, j=1,2$ and $t=1,2, \ldots, T)$. Obviously, $\mathrm{pt}_{\mathrm{t}}\left(\mathrm{Yj}_{\mathrm{j}}, \mathrm{X}_{\mathrm{i}}\right)=1 / \mathrm{pt}\left(\mathrm{X}_{\mathrm{i}}, \mathrm{Y}_{\mathrm{j}}\right)$.

Next we formulate an agent's individual choice problem. Each period t, he must choose a set, $\Phi$ t, of bilateral transactions (at most two) to be carried out simultaneously. Each transaction, $\tau \in \Phi_{\mathrm{t}}$, specifies the goods which are going to be exchanged and the quantities traded (respectively, $\left\{\mathrm{X}_{\mathrm{i}}, \mathrm{X}_{\mathrm{j}}^{\prime}\right\}$ and $\left.\left\{\mathrm{q}^{\tau}\left(\mathrm{X}_{\mathrm{i}}\right), \mathrm{q}^{\tau}\left(\mathrm{X}_{\mathrm{j}}^{\prime}\right)\right\}\right)$. Given the features of the economy we have described, there are several characteristics that these transactions ought to have: (1) they must be informationally feasible. Formally this can be expressed in the following way. For agents of type $A, q^{\tau}\left(C_{1}\right)=0$ and $q^{\tau}\left(A_{1}\right) q^{\tau}(B 1)=0$ (similarly for agents of type B and C). The first part simply means that agents of type A cannot transact with 
good C1. Also, since they transact with agents of type B (or type C), the only high quality good with which they can transact is B (or A, but never both of them together in the same transaction); (2) there cannot be credit in this economy, which implies that the net value of each transaction must be zero. Formally, $p t\left(X_{i}, X_{j}^{\prime}\right) q^{\tau}\left(X_{i}\right)+q^{\tau}\left(X_{j}^{\prime}\right)=0$; (3) a trader's holdings of good $\mathrm{i}$ at the end of period $\mathrm{t}$ must be equal to the holdings he had at the end of period $\mathrm{t}-1$, plus (minus) the quantities he purchased (sold) and minus the quantity he consumed during period t. Formally,

$$
z_{t}\left(X_{i}\right)=z_{t-1}\left(X_{i}\right)+\sum_{\tau \varepsilon \Phi} q^{\tau}\left(X_{i}\right)-e_{t}\left(X_{i}\right)
$$

where $\mathrm{Zt}\left(\mathrm{X}_{\mathrm{i}}\right)$ denotes holdings of good $\mathrm{X}_{\mathrm{i}}$ at the end of period $\mathrm{t}$ and $\mathrm{e}\left(\mathrm{X}_{\mathrm{i}}\right)$ the amount of good $\mathrm{X}_{\mathrm{i}}$ consumed in period t; (4) there cannot be any short sales, that is, traders can only sell goods which they possess. Formally,

$$
z_{t-1}\left(X_{i}\right)+\sum_{\tau \varepsilon \Phi\left(X_{i}\right)} q^{\tau}\left(X_{i}\right)-e_{t}\left(X_{i}\right) \geq 0
$$

where $\Phi_{\mathrm{t}}\left(\mathrm{X}_{\mathrm{i}}\right)=\left\{\tau \in \Phi_{\mathrm{t}} \mid \mathrm{q}^{\tau}\left(\mathrm{X}_{\mathrm{i}}\right)<0\right\}$, that is, transactions which involve sales of good $\mathrm{X}_{\mathrm{i}}$.

Given prices $\{\mathrm{pt}(\cdot, \cdot)\}$, an agent of type A must choose a production/trade/ consumption plan, $\alpha \equiv\left\{\mathrm{z}^{0}(\cdot), \Phi_{\mathrm{t}}, \mathrm{et}(\cdot)\right\} \quad(\mathrm{t}=1, \ldots, \mathrm{T})$, to maximize the following utility function:

$$
\sum_{t=1}^{T}\left(k^{B} e_{t}\left(B_{1}\right)+e_{t}\left(B_{2}\right)\right)
$$

(similarly for agents of type B and C), provided that for all t the restrictions (1)-(4) on 
transactions hold, and, besides (5) $\mathrm{et}(\cdot)$ and $\mathrm{zt}(\cdot)$ are nonnegative for all $\mathrm{t}$; and (6) production is feasible:

$$
h^{A} z_{0}\left(A_{1}\right)+l^{A} z_{0}\left(A_{2}\right) \leq 1, \quad z_{0}\left(B_{i}\right)=z_{0}\left(C_{i}\right)=0
$$

We are now ready to define equilibrium. Equilibrium in this model can be defined as prices $\mathbf{p}\left(\mathrm{X}_{\mathrm{i}}, \mathrm{Y}_{\mathrm{j}}\right)$ for all $\mathrm{t}$ and all pairs of goods $\left\{\mathrm{X}_{\mathrm{i}}, \mathrm{Y}_{\mathrm{j}}\right\}$ and production/trade/consumption plans (one for each agent $\mathrm{h}$ ), $\left\{\boldsymbol{\alpha}^{\mathrm{h}}(\cdot)\right\}$ such that each agent is maximizing his utility subject to (1)-(6) and markets clear (which means that all agents can find a partner for all their transactions). More formally this can be expressed in the following way: for all $\mathrm{t}$, there is a one-to-one correspondence between the set of period $\mathrm{t}$ transactions, $\boldsymbol{\Phi} \mathbf{t}\left(\equiv \cup \operatorname{h} \boldsymbol{\Phi} \mathbf{t}^{\mathbf{h}}\right)$, and itself in which each transaction $\tau$ is paired with its complement $\tau^{\mathrm{c}}$ (obviously, the complement of a transaction is defined as the same transaction with the trading partner's role reversed: the complement of a transaction in which an agent buys $\mathrm{A}_{1}$ and sells $\mathrm{B}_{2}$ is a transaction in which his trading partner buys $\mathrm{B}_{2}$ and sells A1).

\section{Characterization of equilibrium and commodity money}

Lemmas 1 and 2 will help us to identify the (generic) unique equilibrium of the model and the commodity money that characterizes it. We do not provide the proofs of these results and refer the reader to the proofs in BM, which are identical. The only change concerns the different cost structure we assume. 


\section{Lemma 1}

The inequality

$$
\frac{M R S^{X}}{M R T^{X}}\left[\frac{h^{C}\left(M R S^{A} a_{1}+a_{2}\right)}{M R S^{A}}\right]^{T^{*}}\left[\frac{h^{A}\left(M R S^{B} b_{1}+b_{2}\right)}{M R S^{B}}\right]^{T^{*}}\left[\frac{h^{B}\left(M R S^{C} c_{1}+c_{2}\right)}{M R S^{C}}\right]^{T^{*}} \geq 1
$$

holds for $X=A, B, C$, in equilibrium $\left(T^{*}\right.$ is the greatest integer less or equal to $(T+1) / 3$ and $a i, b i$, and ci are per the capita productions of, respectively, $A i, B i$, and $C i)$.

\section{Lemma 2}

If, for given $X \in\{A, B, C\}$, the equilibrium production of $X^{2}$ is positive, then (7) holds with equality for that type of good X.

We are now ready to analyze some features of the equilibrium of the model. In particular, we examine which type of commodity money emerges as the unique medium of exchange. We do this in the following proposition.

\section{Proposition}

Suppose that

$$
\frac{M R S^{A}}{M R T^{A}}<\frac{M R S^{B}}{M R T^{B}}<\frac{M R S^{C}}{M R T^{C}}
$$


Then, in equilibrium, $a^{2}=\left(1 / l^{A}\right)-M R T^{A} a_{1}>0, b^{2}=c^{2}=0, b^{1}=1 / h^{B}, c^{1}=1 / h^{C}$ and al must satisfy

$$
\frac{M R S^{A}}{M R T^{A}}\left[\frac{h^{A}}{M R S^{A}}\left(\left(M R S^{A}-M R T^{A}\right) a_{1}+\frac{1}{l^{A}}\right)\right]^{T^{*}}=1
$$

\section{Proof}

Due to Gresham's law, we know that at least one of $\mathrm{a}^{2}$, $\mathrm{b}^{2}$, or $\mathrm{c}^{2}$ must be positive. Suppose $\mathrm{b}_{2}>0$. This means that (7) holds with equality. Given that $\left(\mathrm{MRS}^{\mathrm{A}} / \mathrm{MRT}^{\mathrm{A}}\right)<$ $\left(\mathrm{MRS}^{\mathrm{B}} / \mathrm{MRT}^{\mathrm{B}}\right)$, it is immediate to show that then Lemma 1 is violated for $\mathrm{X}=\mathrm{A}$. So, it must be that $\mathrm{b}_{2}=0$. The same argument obvioulsy apply to show that $\mathrm{c}_{2}=0$. Then, in equilibrium, $\mathrm{a}^{2}>0$. From Lemma 2 we obtain the condition that a1 should satisfy. QED

\section{Discussion of the results}

The proposition above implies that the unique commodity which in equilibrium will be used as medium of exchange is $\mathrm{A}$, the good for which the ratio $\mathrm{MRS}^{\mathrm{X}} / \mathrm{MRT}^{\mathrm{X}}$ is smallest. This result is merely a generalization of the equivalent result in BM, where $\mathrm{MRT}^{\mathrm{X}}$ is assumed to be the same for all goods. The critical point in the determination of commodity money in both cases is to compare the marginal utility that can be obtained by one unit of labor allocated into a high quality and a low quality version of each type of good. The good for which this ratio is lowest is the unique commodity money in equilibrium, thus guaranteeing that the distortions caused by informational constraints are 
minimized.

Nevertheless, this generalization of the model has paradoxical implications. In order to see them, let us compare two almost identical economies (denoted by 1 and 2 ) which only differ in the cost of producing low quality goods of type A. Suppose $1_{1}^{\mathrm{A}}<1_{2}^{\mathrm{A}}$ (so, $\mathrm{MRT}_{1}^{\mathrm{A}}>\mathrm{MRT}_{2}^{\mathrm{A}}$ ) such that now $\left(\mathrm{MRS}^{\mathrm{A}} / \mathrm{MRT}_{2}^{\mathrm{A}}\right)>\left(\mathrm{MRS}^{\mathrm{B}} / \mathrm{MRT}^{\mathrm{B}}\right)>\left(\mathrm{MRS}^{\mathrm{A}} / \mathrm{MRT}_{1}^{\mathrm{A}}\right)$. The proposition above implies that good A (e.g. gold) will be used as commodity money in economy 1 , but not in economy 2 . Then, lower production costs of low quality gold coins may induce gold into being used as medium of exchange. This clearly sounds paradoxical and contradictory with the notion that money should be a good hard to fake. How is this paradox to be explained? The crucial point to be taken into account is that, due to the assumptions about restrictions on trader's information, there are not "real" goods circulating as money in this environment. In fact, only low quality goods are media of exchange. Consequently, it is less surprising that making fake gold coins more expensive to produce may drive them out of circulation as money. It is not gold, but rather counterfeit gold that becomes the medium of exchange when counterfeiting is easy. In equilibrium, commodity money is the low quality version of the good for which the ratio $\mathrm{MRS}^{\mathrm{X}} / \mathrm{MRT}^{\mathrm{X}}$ is smallest. In $\mathrm{BM}$, where $\mathrm{MRT}^{\mathrm{X}}$ is identical for all goods, this can be interpreted rather intuitively as the good for which $\mathrm{MRS}^{\mathrm{X}}$ is smallest, that is, the one for which differences in quality that are undetectable are particularly small. Once the model is generalized to allow for different MRT's, the interpretation becomes less convincing. A low ratio $\mathrm{MRS}^{\mathrm{X}} / \mathrm{MRT}^{\mathrm{X}}$ can be caused by a low value of $\mathrm{MRS}^{\mathrm{X}}$, but also by high values of $\mathrm{MRT}^{\mathrm{X}}$. The latter means that low quality versions of good $\mathrm{X}$ (the medium of exchange) 
are relatively much cheaper to produce than high quality versions, but this a result that goes against general economic conventions about the desirable properties of money.

This same reason lies behind the second paradoxical result we highlight. Due to the difference in the production technology between the two economies, the distortions imposed by the informational constraints are larger for economy 2 . This is because these distortions are due to the production of low quality goods, which are socially useful to play the role of means of exchange in the presence of problems of asymmetric information. If low quality goods are more expensive to produce, then society will have to spend extra resources to produce those "lemons" and, consequently, allocative distortions due to asymmetric information will become larger. The result can be summarized as follows: the economy in which production of low quality counterfeits is easier will bear a lower burden, in terms of allocative efficiency, due to the generic asymmetric information problems assumed in the model. 


\title{
Reference
}

BANERJEe, A.V. AND MASKIN E.S.: "A Walrasian Theory of Money and Barter" Quarterly Journal of Economics 111, 955-1005 (1996).

\section{Notes}

\begin{abstract}
${ }^{1}$ Alternatively, this could be interpreted as differences in the technology used to detect "lemons", which make undistinguishable low quality goods more or less difficult to produce.
\end{abstract}

